\title{
SHAXSIY YOZISHMALARDA VULGAR SO`ZLAR VA LINGVISTIK EKSPERTIZA
}

\section{To'rayeva Dildora Anvarovna}

A.Navoiy nomidagi Toshkent davlat o'zbek tili va adabiyoti universiteti "Amaliy tilshunoslik va lingvodidaktika" kafedrasi o"qituvchisi dildoratorayeva9@gmail.com

Annotatsiya. Ushbu maqolada o'zbek tilidagi matnlardagi noadabiy leksik unsurlar haqida ma'lumot berilgan. Lingvistik ekspertiza tushunchasi, uning turli sohalardagi va insoniyat oldidagi ahamiyati, matn tahlilida ekspert oldidagi vazifalar haqida mulohazalar yuritilgan.

Kalit so'zlar: Vulgar so'zlar, varvar so'zlar, lingvistik ekpertiza,yuristlingvistika, lingvistik ekspertiza usullari, aniq fakt, dalil.

Annotation. This article provides information on non-literary lexical elements in Uzbek texts. The concept of linguistic expertise, its importance in various fields and to humanity, the tasks of the expert in the analysis of the text are discussed.

Keywords: Vulgar words, barbaric words, linguistic expertise, methods of linguistic expertise, concrete fact, evidence.

Аннотация. Эта статья предоставляет информацию о иррациональных лексических элементах в узбекских текстах. Концепция лингвистической экспертизы, его важность в различных областях и человечности, текстовый анализ обсуждает задачи эксперта.

Ключевые слова: Паразитарные слова, словарные слова, методы переписки, лингвистические методы обследования, доказательства.

Xalq orasida umumiy me'yorlardan chetga chiqadigan noadabiy leksik unsurlardan biri borki, u so'zlovchining nutq vaziyati, maqsadi va psixik holatiga ko'ra namoyon bo'ladi. Bu vulgarizm (lot. Vulganis - dag'al, qo'pol so'z) bo'lib, adabiy tilda ishlatilmaydigan qo 'pol so'zlar, til me'yorlariga to ' $\mathrm{g}$ 'ri kelmaydigan, noto'g'ri tuzilgan jumlalar[3]. Og'zaki va yozma nutqda ba'zan uchrab turadigan so'kinish haqorat, qarg'ish so 'zlari jamiyatimizning axloq me'yorlariga zid hodisa sifatida nutqimiz sofligiga ham salbiy ta’sir qiladi. 
Vulgarizmlarni ma'no jihatidan ikki guruhga bo'lish mumkin:

1. So'kinish so'zlari.

2. Qarg'ishlar.

So'kinch so`zlarning qo'llanish darajasiga ko`ra 3 guruhga bo'lish mumkin:

1) Haqorat so 'kinch so'zlar;

2) Qo'pol so'kinch so'zlar;

3) O'ta qo'pol so'kinch so'zlar.

So'kinish so'zlari insonning aynan xarakter-xususiyatidan kelib chiqib aytilishi mumkin, yoki unga nisbatan noo'rin tarzda amalga oshirilishi mumkin. Aynan lingvistik ekspertizda shu nuqtai-nazardan yondashgan holda jabrlanuvchiga nisbatan nohaq ishlatilgan so'kinish so'zlarigina haqorat so' $\mathrm{z}$ sanaladi. Masalan, o'g'ri nomini bersak-u, asli $\mathrm{u}$, umuman, o'g'rilikka qo' 1 urmagan bo'lsa, yoki keng omma oldida sha'niga, g'ururiga tegadigan haqorat so 'zlaridan foydalansa, bu o'rinda jinoiy ish qo' $z g^{\text {' }}$ atilishi mumkin.

Lingvistik ekspertiza-mustaqil sud ekspertizasi, nutq ma'lumotlari (har qanday moddiy vositada qayd etilgan xabar)ni lingvistik o'rganish bo'yicha olib boriladigan tahlil jarayoni[Ярощук, 2020:8]. Ushbu faoliyat natijalari jinoiy, fuqarolik yoki arbitraj jarayoni doirasida qat'iy tartibga solinadi va tilshunos ekspert (yoki ekspert tilshunoslar komissiyasi) tomonidan yozma xulosa bilan rasmiylashtiriladi. Ushbu xulosalar tilshunoslikda maxsus bilimlarni qo'llashni talab qiladigan masalalarni $\mathrm{o}^{6} \mathrm{z}$ ichiga oladi. Bu sud jarayonida muammoli shaxsiy yozishmalar tahlilidagi farazlar, taxminlarni oldini oladi va individual baholashniong aniq mezonini belgilab beradi.

Lingvistik ekspertiza olib borishdan maqsad maxsus filologik bilimlar asosida ma'lum bir sohadagi muammoli matnlardagi faktlar, vaziyatlarni aniqlash. Shu o'rinda muammoli matn nima degan o'rinli savol tug'iladi. Bahs-munozaraga sababchi bo'ladigan matnlar sirasiga quyidagilar kiradi:

a) hujjat;

b) gazetada e'lon qilingan maqola;

c) teleko'rsatuv;

d) firma nomi;

e) tovar belgisi.

Avval munozarali matn turi aniqlab olinadi, so"ng tilshunos tomonidan uning janr, kompozitsion, fonetik, leksik-grammatik ifoda shakllari o'rganiladi. Yuqorida aytganimizdek, agar konfliktli matnda haqorat so'zlari bo'lsa, avvalo, haqorat so'zlarning ifoda darajasi aniqlab olinadi, Bu lingvistik ekspertiza uchun 
muhim. Bunday so' $z$ qatlami hamma vaqt ham o'z semasini namoyon etmasligi mumkin. Masalan, Voy jinnivoyim o'zimning, qachom katta bo'lasana...(So'zlashuv nutqidan. 14.04.21.) $\mathrm{Bu}$ o'rinda haqorat so'zi shaxsni obro'sizlantirish maqsadidan yiroq. Haqorat so'zining darajasi nutqiy vaziyatga ko'ra aniqlanadi. Tilshunos aynan haqorat so'zning semantic mohiyatini aniqlash bilan vaziyatga oydinlik kirita oladi. Masalan, Odamlar ishini qilib tushlikka chiqdi. Sen hali ham bolishdan boshingni uzmabsan. Ahmoq! ("Ufq" S.A.) Voydod, sho'rimiz qurib qoldi. Sharmanda bo'ldik, qizni olib qochdi. Bir yashshamagur, qirchiningdan qiyilgur qizni olib qochdi.("Ufq"S.A.) Personajning xatti-harakatlari $\mathrm{O}^{\prime} \mathrm{z}$ shaxsiyatiga nisbatan haqorat so'zlarining ishlatilishiga sabab bo'lyapdi. So'kinch so'zlarning darajasini aniqlash uchun quyidagi tasnif ancha qo'1 keladi:

a) axloqiy madaniyatli - haromi, la'nati kabi;

b) axloqiy madaniyatsiz - jalab, mochaxar kabi;

v) hayvon nomlari bilan bog'liq - chivin, it, eshak, ho'kiz kabi;

So'kinish so'zlarining bu uch turi ham nutqiy vaziyat va maqsad bilan bog'liq bo'lib, so'z sememasining kuchli-kuchsizligi maslaga oydinlik kiritadi. Zero, jamoa oldida insonni obro'sizlantirish, uning shaxsiyatiga mos kelmaydigan so'zlardan nohaq foydalanish, uning mansabi, sha'ni, g'ururiga dahl qilish kabi vaziyatlar uchun tilshunos ekspert tahlili muhim sanaladi. Va bu ish uchun jinoiy javobgarlik qo'zg'atiladi. Avvalo, ekspert huquqbuzarlikning turiga ko'ra, matnni tahlil qilishi va ma'lum xulolasini berishi uchun ikki yondashuv mavjud:

1) asosiy faktlar;

2) dalillarga asoslangan ma'lumotlar.

Asosiy fakt - uning barcha elementlarida jinoyat ishi borligi yoki yo'qligi va dalil bu boshqa faktlar bilan birgalikda asosiy fakt haqida xulosa chiqarish mumkin bo'lgan haqiqatdir[Белкин,1966:19]. Har qanday jinoyatning obyektiv va subyektiv tomoni bo'ladi. Jinoyatning obyektiv jihatini yoritishda asosiy va dalillarga asoslangan ma'lumotlar muhim hisoblanadi. Negaki sud hakami ekspertga mavjud ishda jinoyat bormi, yo'qmi deb savol bersa, asosiy fakt haqida so'ragan bo'ladi. Agar matndagi so'zlarning semantikasi, garammatikasi, fonetik xususiyatlari haqida so'rasa, endi ekspert dalillardan kelib chiqib javob beradi. Bu ekspertning tahlil jarayoniga obyektiv aniqlik kiritadi. Masalan, munozarali shaxsiy yozishmada haqoratomuz so'zlar mavjudligi yoki yo'qligi asosiy fakt bo'Isa, ularning kimga qaratilganligi, mazmuniy darajasi, leksik qiymati bular dalil ma'lumotlar sanaladi. 
Jinoyatning subyektiv tomonida esa qonunbuzarning niyati aniqlanadi. Masalan, ma'ruzachi yolg'on gapirmayotgandek tutadi, shunda uning kommunikativ niyatini aniqlash muhim sanaladi. Yoinki, haqiqatni ro'y-rost da'vo qiladi, yo haqiqatni da'vo qilayotgandek o'zini tutadi. Bularning barida so'zlovchining niyati, maqsadini aniqlash lozim. Jinoyatchining niyat, maqsadini aniqlash, yoki matnga kiritilmagan faktlarni tahlil qilish, yoinki jinoyatning sababini topish - bularning bari ekspertning vakolatiga kirmaydi. Matnning lingvistik ekspertizasini ishlab chiqishda ekspertning vakolatiga matnning ma'nosini, ya'ni unda nima deyilganini belgilash kiradi. U muallifning matnida ifodalangan niyat va maqsadini tahlil qiladi. Avvalo, dalil sifatida olingan matndagi so 'zlovchining niyati sud tomonidan aniqlanadi, lingvist esa masalaga xususiy yondashib lingvistik asoslarini belgilab beradi.

\section{Foydalanilgan adabiyotlar:}

1. Белкин Р. С. Собирание, исследование и оценка доказательств. М., 1966. C. 19.

2. Ярощук И.А., Жукова Н.А., Долженко Н.И. . Лингвистическая экспертиза : учебное пособие / - Белгород : ИД «БелГУ» НИУ «БелГУ», 2020. $-8 \mathrm{c}$.

3. https://uz.wiktionary.org/wiki/vulgarizm 